\title{
Die Bedeutung von Stigmatisierungsprozessen bei Essstörungen und Übergewicht
}

Ulrich Hagenah

\section{Einleitung}

Die Adipositas hat in vielen Ländern der Erde mittlerweile epidemische Ausmaße angenommen und die Unterernährung in ihrer medizinischen Bedeutung überrundet. ${ }^{1}$ Die hohen körperlichen und psychischen Belastungen der Betroffenen werden durch die durch Übergewicht bedingten Folgeerkrankungen häufig verstärkt. Im Kontrast zu dieser Entwicklung steht ein extrem schlankes Schönheitsideal in diesen Gesellschaften, dessen Bedeutung sich in Kernsymptomen moderner Essstörungen wie Anorexia und Bulimia nervosa widerspiegelt. Die Anorexia nervosa stellt inzwischen die dritthäufigste chronische Erkrankung bei Adoleszenten dar und weist gleichzeitig die höchste Mortalität aller psychiatrischen Erkrankungen auf. ${ }^{2}$ Essstörungen führen nicht nur zu zahlreichen körperlichen und psychischen Störungen, sondern zusätzlich auch zu erheblichen psychosozialen Belastungen und Beeinträchtigungen der Lebensqualität, nicht zuletzt im Zusammenhang mit Stigmatisierungsprozessen, denen Betroffene und ihre Angehörigen häufig ausgesetzt sind.

\section{Essstörungen}

Nicht nur aus dem alten Rom, sondern auch in griechischen und ägyptischen Überlieferungen wird absichtlich herbeigeführtes Erbrechen als kulturell

1 Hebebrand et al. (2004).

2 Nicholls/Viner (2005). 
etablierte Verhaltensweise, z. B. mit dem Ziel, sich anschließend weiter der Völlerei widmen zu können, berichtet. ${ }^{3}$ Auch Fälle extremen Fastens finden sich bereits in historischen Schilderungen, häufig im Zusammenhang mit religiösen Motiven. Die Lebens- und Leidensgeschichte der $\mathrm{Hl}$. Katharina von Siena (1347-1380), die bereits im Alter von sieben Jahren zunächst auf Fleisch verzichtet haben soll ${ }^{4}$ und später wahrscheinlich an den Folgen des Hungerns starb, erinnert in vielen Aspekten an das Krankheitsbild einer heutigen Anorexia nervosa. Allerdings fehlt in derartigen historischen Schilderungen die für die modernen Essstörungen typische Überbewertung von Gewicht und Figur.

Kernsymptome der Anorexia nervosa sind eine exzessive Beschäftigung mit dem Körperbild und eine krankhafte Furcht vor einer Gewichtszunahme (Gewichtsphobie). Der Gewichtsverlust wird durch ausgeprägte, selektive Nahrungsrestriktion, z.T. auch durch Erbrechen und Medikamentenabusus, erreicht. Selbst bei erheblicher Abmagerung (Kachexie) besteht das Streben, noch dünner, noch schlanker zu werden, fort. Eine 14-jährige Jugendliche beschrieb diesen Prozess während der stationären Behandlung in der Klinik: „Am Anfang war mein Ziel, wieder unter $50 \mathrm{~kg}$ zu wiegen. Als ich nur noch 40 $\mathrm{kg}$ wog, wollte ich unbedingt $35 \mathrm{~kg}$ wiegen ... Momentan ist mein Ziel, unter $28 \mathrm{~kg}$ zu wiegen ..."Viele Patientinnen zeigen trotz der schlechten körperlichen Verfassung ein extremes Ausmaß an körperlicher Aktivität. In vielen Fällen ist eine Krankheitseinsicht bei den überwiegend weiblichen Patientinnen nicht erkennbar.

Pathognomonisches Merkmal der Bulimia nervosa sind Essattacken. Weitere Kriterien nach ICD-10 sind zusätzliche gewichtsreduzierende Maßnahmen (z. B. Erbrechen, Abführmittelmissbrauch, Sport, Fasten), eine Gewichtsphobie sowie eine eventuell in der Vorgeschichte bestehende Magersucht. Ungefähr 5-10\% aller Menschen mit Adipositas erfüllen die Forschungskriterien für die so genannte Binge Eating-Störung, die primär durch Essattacken bei fehlender Gegenregulation (z. B. Fasten, Erbrechen, Abführmittelabusus)gekennzeichnet ist.

Die Punktprävalenz bei Jugendlichen und jungen Frauen liegt für die Anorexia nervosa zwischen 0,2 und o, $8 \%$, für die Bulimia nervosa zwischen 1-2\%. Seit den 193oer Jahren ist eine kontinuierliche Zunahme der Anorexia nervosa in den westlichen Industrienationen in der Altersgruppe der 15- bis 24-jährigen Frauen dokumentiert. ${ }^{6}$ Für die Bulimia nervosa scheinen die Prävalenzraten nach deutlichen Anstieg bis in die 1990er Jahre zuletzt wieder zu sinken. ${ }^{7}$ Konsistent wird in der Literatur ein Überwiegen des weiblichen Geschlechts von etwa zehn zu eins (Anorexia nervosa) beziehungsweise bis zu dreißig zu eins (Bulimia nervosa) mitgeteilt. ${ }^{8}$ Während lange angenommen wurde, dass

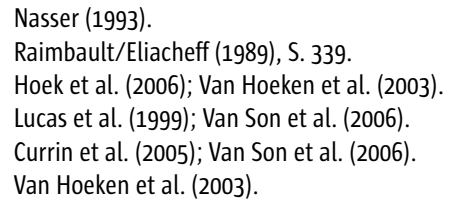


Anorexia und Bulimia nervosa in erster Linie in den westlichen Industrienationen auftreten, ist mittlerweile deutlich geworden, dass auch in anderen Kulturen und ethnischen Gruppen das Erkrankungsrisiko für diese Essstörungen ansteigt. ${ }^{9}$

Die Genese von Essstörungen wird heute multifaktoriell im Sinne eines Wechselspiels zwischen biologischen, soziokulturellen und individuellen Risikofaktoren betrachtet. Während genetische Faktoren wahrscheinlich zu einer erhöhten Vulnerabilität gegenüber Nahrungsrestriktion beitragen, scheinen soziokulturelle Einflüsse zu der Zunahme der Prävalenz in den letzten Jahrzehnten beigetragen zu haben. Als individuelle Risikofaktoren für die Entwicklung einer Essstörung wurden ein erniedrigtes Selbstwertgefühl und weniger konsistent - ein hoher Grad an Perfektionismus ermittelt. ${ }^{10}$ Prospektiv erhobene Daten verweisen auf die Bedeutung von restriktivem Essen (Diät-Halten) für die Entwicklung einer Essstörung. ${ }^{11}$ Dem extremen Schlankheitsideal in westlichen Industrienationen ist bereits früh eine ätiologisch bedeutsame Rolle zugeschrieben worden. ${ }^{12}$

Die Einordnung von Übergewicht und Adipositas als Krankheit wird aus unterschiedlichen Perspektiven kontrovers diskutiert. ${ }^{13}$ In Deutschland sind mittlerweile ungefähr $50 \%$ der Erwachsenen als übergewichtig bzw. $20 \%$ als adipös, d.h. fettsüchtig, eingestuft. ${ }^{14}$ Medienberichte mit Schlagzeilen „Deutschland ist in Europa Dickenland Nummer 1“, bzw. „Deutschland ist in der EU am fettesten“ im Zusammenhang mit einer Studie der International Association for the Study of Obesity kommen sogar zu dem Schluss: „In Deutschland sind $75 \%$ der Männer und 59 \% der Frauen zu dick" und münden in die plakativ aufgemachte Forderung des deutschen Verbraucherministers Horst Seehofer: „Deutsche, speckt ab!“ ${ }^{15}$ Kritisch ist bei derartigen Studien die Gleichsetzung eines Body-Mass-Index (BMI) von mehr als $25 \mathrm{~kg} / \mathrm{m}^{2}$ unabhängig von anderen Faktoren als Übergewicht. Nach diesen Kriterien wäre beispielsweise der langjährige Nationaltorwart Oliver Kahn laut Angaben zu seinen Körpermaßen auf der Homepage des FC Bayern München (1,88 m, 91 kg) mit einem BMI von 25,7 $\mathrm{kg} / \mathrm{m}^{2}$ übergewichtig! Neben dem Ausmaß des Übergewichts, welches über den Body-Mass-Index erfasst wird, scheint vor allem die viszerale Fettmasse besonders eng mit kardiovaskulären Risikofaktoren und Komplikationen zu korrelieren. ${ }^{16}$

Als ätiologisch bedeutsam für die Entstehung von Übergewicht und Adipositas wird das Wechselspiel zwischen Genvarianten, die eine Energiespeicherung begünstigen (,thrifty genotype“-Hypothese, „thrifty“ = „sparsam“) mit einem in modernen Industrienationen und Schwellenländern leicht verfügbaren Angebot an energiereichen und schmackhaften Nahrungsmitteln

\footnotetext{
9 Makino et al. (2004), Gunewardene et al. (2001).

10 Fairburn/Harrison (2003); Jacobi et al. (2004).

11 Patton et al. (1990).

12 Bruch (1962).

13 Hebebrand et al. (2004).

14 Bergmann/Mensink (1999).

15 Der Stern, 20. April 2007.

16 Deutsche Adipositas-Gesellschaft (2007).
} 
sowie einer parallel zu beobachtenden Abnahme der körperlichen Aktivität angesehen. ${ }^{17}$ Zusätzlich werden bei der Entstehung von Übergewicht psychologische Mechanismen diskutiert, für die vor allem das Belohnungssystem („Reward-System“) eine bedeutende Rolle spielt. Die Aufnahme von Nahrung führt zu einer erhöhten Dopamin-Konzentrationen im Nucleus accumbens (NA), die mit einem ausgeprägten Lustgefühl verbunden ist. ${ }^{18}$ Cenetisch veränderte Mäuse, bei denen die Dopamin-Synthese ausgeschaltet wurde, zeigen kein Interesse an Nahrung und sterben. ${ }^{19}$ Vereinfacht ausgedrückt essen wir, um das Belohnungssystem zu aktivieren, die Beseitigung von Hunger ist in neurobiologischer Hinsicht ein Nebeneffekt. Besonders deutlich wird dies beim so genannten „Frust-Essen“. Zusätzlich lässt sich Appetit konditionieren. Ratten, die nach Hungern Futter in Kombination mit einem akustischen Reiz erhielten, fraßen, wenn sie satt waren, mehr wenn ihnen das Futter gleichzeitig mit demselben Stimulus angeboten wurde. ${ }^{20}$ Die Möglichkeit der Appetit-Konditionierung wird durch die Werbung der Nahrungsmittelindustrie genutzt. Eine Hauptzielgruppe sind Kinder. Fernsehsendungen für diese Altersgruppe werden durch Werbespots für hochkalorische Fast-Food-Produkte und Softdrinks unterbrochen. Derartige Werbung ist erfolgreich: nach Exposition kaufen Kinder selbst häufiger entsprechende Produkte, fordern diese von ihren Eltern ein, und Eltern kaufen diese für ihre Kinder. ${ }^{21}$

Die durch Adipositas und deren Folgen hervorgerufenen Kosten werden mittlerweile in Deutschland auf $6 \%$ der Krankheitskosten geschätzt. ${ }^{22}$

\section{Stigmatisierung}

Mit dem Begriff Stigma (abgeleitet vom griechischen BegriffStigma: Punkt, Brandmal, Stich) - von Goffman (1963) zunächst in die soziologische Diskussion eingeführt - wird die Verknüpfung eines bestimmten Merkmals (z. B. „psychisch krank“) einer Person oder Gruppe mit einem negativen sozialen Stereotyp oder Vorurteil (,ist grundsätzlich gefährlich“) bezeichnet. ${ }^{23}$ Hiermit verbunden ist ein Statusverlust des Stigmatisierten, der zu Benachteiligung und Abwertung (Diskriminierung) führt. Betroffen sind häufig so genannte Randgruppen (z.B. Migranten, Arbeitslose, Prostituierte, Homosexuelle, Menschen mit körperlichen Erkrankungen oder Behinderungen). Die Stigmatisierung von psychisch kranken Menschen hat eine lange Tradition. So war beispielsweise im mittelalterlichen Köln für „Besessene“ die sogenannte Kreuztonsur vorgeschrieben, bei der lediglich zwei einander überschneidenden Haarstreifen auf dem Haupt verblieben. ${ }^{24}$ Geistesgestörte ,galten gleichsam als Dauerbesesse-

17 Hebebrand et al. (2004).

18 Saper/Chou/Elmquist (2002).

19 Szczypka et al. (2001).

20 Petrovich et al. (2005)

21 Übersichten bei: Coon/Tucker (2002); The Henry I Kaiser Family Foundation (2004).

22 Pressemitteilung der Bundesärztekammer vom 12.01.2007.

23 Link/Phelan (2001).

24 Müller (1996), S. 56. 
ne [...], mit ihrem gebrochenen Stammeln und den verzerrten Zügen ihres Verhaltens nur das Abbild des Ungeistes, der sie beherrschte. “25

Stigma und Diskriminierung haben einen komplexen, biopsychosozialen Hintergrund. ${ }^{26}$ Das menschliche Gehirn scheint negative Erfahrungen (die seltener sind als neutrale oder positive Erlebnisse) tendenziell eher mit anderen selteneren Objekten zu verknüpfen, zum Beispiel mit Angehörigen von Minderheiten. ${ }^{27}$ Die Bildung von Kategorien und Stereotypen ermöglicht es, auf eingehende Informationen schneller zu reagieren. Stigmatisierung als Taktik für Überleben und Fortpflanzung scheint unter evolutionären Aspekten mit Vorteilen verbunden gewesen zu sein. Unter schwierigen wirtschaftlichen Zeiten kann Stigmatisierung von Mitbewerbern auch heutzutage ein wirkungsvolles Mittel sein, z. B. im Kampf um Arbeitsplätze. Stigmatisierung dient in psychologischer Sicht der Selbstwerterhöhung, da durch den Vergleich mit anderen Personen, denen es schlechter geht, eigene Eigenschaften positiver erlebt werden. Dies gilt auch für soziale Gruppen: So waren z. B. Probanden einer Cruppe, in denen Belohnungen für die Durchführung einer Aufgabe gerecht verteilt wurden, weniger zufrieden als diejenigen einer Vergleichsgruppe, in denen eine Person benachteiligt wurde, auch wenn alle Teilnehmer die Ungerechtigkeit der Situation erkannt hatten. ${ }^{28}$

Die Folgen von Stigmatisierung und Diskriminierungen psychisch kranker Menschen zeigen sich im konkreten Alltagsleben, z. B. durch Benachteiligung bei der Arbeits- und Wohnungssuche. Schizophren erkrankte Menschen werden von ihrer Umwelt pauschalierend als überdurchschnittlich aggressiv und gewalttätig, Menschen mit Depressionen und Suchterkrankungen als „selber Schuld an ihrer Erkrankung“ eingestuft. Im Hinblick auf die negativen Folgen von Stigmatisierung sind nicht nur die von der Umwelt ausgehenden Prozesse (öffentliche Stigmatisierung), sondern auch die Internalisierung diskriminierender Stereotype durch die Betroffenen selbst (Selbststigmatisierung) zu beachten. Diese spielt wahrscheinlich bei psychisch erkrankten Menschen eine noch bedeutsamere Rolle als die öffentliche Stigmatisierung. Selbststigmatisierung, z. B. die Annahme, dass Ärzte auf eine psychische Erkrankung negativ reagieren könnten, wenn man bei ihnen Hilfe sucht, kann die Schwelle zur Inanspruchnahme von Hilfe durch das Gesundheitssystem erhöhen. ${ }^{29}$ Bei einer Neuerkrankung werden negative Konzepte über psychische Erkrankungen auf sich selbst bezogen, was zur Verringerung des Selbstwertgefühls führen kann und auf diese Weise oft einen negativen Einfluss auf den Krankheitsverlauf zur Folge hat. Der soziale Rückzug von Menschen mit psychischen Erkrankungen wird durch das Bemühen der Betroffenen verstärkt, Symptome der Erkrankung vor Freunden und Arbeitskollegen zu verheimlichen. Auf diese Weise reduzieren sich die Chancen auf soziale Unterstützung bei der Bewältigung von Alltag und Erkrankung weiter.

25 Müller (1996), S. 34.

26 Haghighat (2001).

27 Z. B. Stroessner/Mackie (1993).

28 Brickman (1975).

29 Barney et al. (2006). 
Das Stigma psychischer Erkrankungen lässt sich auch unter Ärzten in einer oftmals skeptisch-negativistischen Einstellung gegenüber psychischen Erkrankungen und ihren Therapiemöglichkeiten, die aktuelle positive Entwicklungen in der Behandlung psychischer Erkrankungen außer Acht lässt, aufzeigen. ${ }^{30}$ Die zunehmend erkannte Bedeutung von Stigmatisierung spiegelt sich in den vergangenen Jahren in einer deutlichen Zunahme der veröffentlichten Arbeiten wider. Zwischen 1998 (47 Artikel) und 2006 (215 Artikel) wuchs die Anzahl der in Medline registrierten Arbeiten zu diesem Bereich um fast das fünffache (Suchstrategie: „stigma“ + „social“).

\section{Stigmatisierung im Zusammenhang mit Übergewicht und Adipositas}

Adipöse Menschen werden oft schon als Kinder und Jugendliche von Gleichaltrigen gehänselt und von Lehrern bloßgestellt (oft z. B. im Sportunterricht), haben seltener einen Schulabschluss, sind unter Studenten unterrepräsentiert, heiraten seltener, erzielen später ein geringeres Einkommen ${ }^{31}$ und sind auch mit anderen Benachteiligungen auf dem Arbeitsmarkt konfrontiert. Dies gilt besonders für übergewichtige Frauen. ${ }^{32}$ Obwohl die Erkenntnisse zur Genetik der Adipositas inzwischen verdeutlichen, dass ein erheblicher Anteil der Ursachenfaktoren willentlich nicht einfach beeinflussbar ist, wird die Adipositas in der Öffentlichkeit, aber auch in Medizin und Psychologie, immer noch häufig als durch die betroffene Person bei entsprechender Willensanstrengung leicht veränderbar dargestellt. Da die meisten Betroffenen bei diesen Versuchen aber langfristig scheitern, resultieren Schuldgefühle, sozialer Rückzug und Stigmatisierung als negative Folgen. ${ }^{33}$

Das Image von Übergewichtigen ist mit Attributen wie Undiszipliniertheit, Schlampigkeit und Unzuverlässigkeit assoziiert. ${ }^{34}$ Stigmatisierende Gleichsetzungen von „übergewichtig“ mit „mangelnder Selbstkontrolle“ oder „Faulheit“ sind auch bei professionellen Helfern im Gesundheitssystem häufig anzutreffen. ${ }^{35}$ Positive Bewertungen von Übergewicht und Adipositas - in früheren Zeiten und zum Teil heute noch in anderen Kulturen wurde bzw. wird Übergewicht als Zeichen für Wohlstand interpretiert - haben in den vergangenen 40 Jahren erkennbar abgenommen. Während in einer ersten Erhebung 1971 noch mehr als die Hälfte der befragten Teilnehmer die Silhouette eines dicken bzw. sehr dicken Menschen mit Qualitäten wie „Verträglichkeit“ $(64 \%)$ und „Lebensfreude“ (50\%) verbanden, taten dies 1979 nur noch $28 \%$ beziehungsweise $9 \%$. Bezüglich der Frage: „Mit wem möchten Sie gerne befreundet sein?" reduzierte sich zwischen 1971 und 1979 der Anteil der Teilnehmer, die sich für eine dieser beiden Silhouetten entschied, von $40 \%$ auf $3 \%{ }^{36}$

\footnotetext{
30 Gaebel et al. (2004).

31 Gortmarker (1993).

32 Roehling et al. (1999); Cawley/Danziger (2004).

33 Hebebrand et al. (2004).

34 Robert Koch-Institut/Statistisches Bundesamt (2005).

35 Price et al. (1987); Myers/Rosen (1999).

36 Westenhöfer/Pudel (1990).
} 
Dass Stigmatisierungsprozesse bei Übergewicht bereits im Kindesalter eine Rolle spielen, ist seit den 196oer Jahren gut belegt. Negative Einstellungen gegenüber übergewichtigen Kinder konnten bei Kindern im Alter von drei Jahren ermittelt werden, die anhand von Bildern pummelige Kinder zum Beispiel als unbeliebte Spielkameraden bewerteten. ${ }^{37}$ Derart frühe stigmatisierende Erfahrungen übergewichtiger Kinder tragen möglicherweise dazu bei, dass diese häufiger ein niedrigeres Selbstwertgefühl und größere Scham zeigen und häufiger über erlebte Sticheleien und Erniedrigungen berichten als nicht übergewichtige Gleichaltrige. ${ }^{38}$ Übergewichtige Kinder fehlen häufiger in der Schule, was zum Teil auf ein erhöhtes Risiko für somatische Erkrankungen zurückgeführt werden kann, andererseits aber auch mit einer erhöhten Rate an komorbiden psychischen Störungen und einer schlechteren sozialen Integration in Gleichaltrigengruppen zusammenhängen könnte. Trotz eines deutlichen Anstiegs übergewichtiger Kinder ${ }^{39}$ konnte zwischen 1961 und 2001 eine Zunahme der negativen Einstellungen gegenüber übergewichtigen Kindern nachgewiesen werden, z. B. bei Fünft- und Sechstklässlern an amerikanischen Schulen. ${ }^{40}$ Abwertende Kommentare erleben übergewichtige Kinder häufig auch innerhalb der eigenen Familien durch Eltern und Geschwisterkinder. ${ }^{41}$

Die Annahme einer organischen Ätiologie des Übergewichts kann möglicherweise Stigmatisierung verhindern oder reduzieren. So beurteilten amerikanische High-School-Schülerinnen übergewichtige gegenüber normalgewichtigen Personen in einer Videoaufnahme vor allem dann als „maßlos“ und „undiszipliniert“, wenn ihnen nicht vorher als Ursache des Übergewichts eine Drüsenerkrankung erklärt worden war. ${ }^{42}$ Dagegen wird eine Stigmatisierung von Übergewicht durch eigenes Übergewicht der Befragten nicht reduziert. ${ }^{43}$

Stigmatisierungen treffen nicht nur die Kinder und Jugendlichen selbst, sondern auch deren Familien. Im Hinblick auf die Prävention der Adipositas herrscht mittlerweile Konsens, dass diese bereits bei Kindern ansetzen muss, und ein effektives Betreuungsprogramm zu einer langfristigen Gewichtsreduktion bei Kindern besonders dann führt, wenn die Eltern der Kinder als Zielgruppe für Verhaltensänderungen einbezogen sind. ${ }^{44}$ Dennoch beklagen viele Eltern, dass sie in ihren Sorgen bezüglich eines sich entwickelnden Übergewichts ihrer Kinder von Ärzten nicht ernst genommen oder hingehalten gefühlt hatten oder ihnen Vorwürfe, am Übergewicht ihres Kindes schuld zu sein, gemacht worden waren. ${ }^{45}$ Möglicherweise tragen Bildungs- und Schichtunterschiede zwischen professionellen Helfern und den Familien mit überge-

\footnotetext{
37 Cramer/Steinwert (1998).

38 Pierce/Wardle (1997).

39 Ogden et al. (2002); Herpertz-Dahlmann et al. (2003).

40 Latner/Stunkard (2003).

41 Taylor et al. (2006).

42 Dejong (1993).

43 Latner/Stunkard/Wilson (2005).

44 Epstein et al. (1994).

45 Edmunds (2005).
} 
wichtigen Kindern zusätzlich zu diesen Schwierigkeiten bei, da diese Faktoren stark mit dem kindlichen Übergewicht assoziiert sind. ${ }^{46}$

Im Zusammenhang mit den volkswirtschaftlichen Belastungen durch die Adipositas und ihre Folgen zeigt sich in den letzten Jahren in der Öffentlichkeitsarbeit eine deutliche Tendenz, neben Aufklärung über die gesundheitlichen Folgen des Übergewichts eine vermeintliche Eigenverantwortung der Betroffenen zu unterstreichen, die aber durch wissenschaftliche Daten nicht belegbar ist ${ }^{47}$ und die möglicherweise das Risiko öffentlicher Stigmatisierung noch vergrößert.

Im Kontrast zum zunehmenden Druck auf die Betroffenen, ihr Gewicht zu reduzieren, steht die ernüchternde Erkenntnis, dass derzeitige, konventionelle Therapieverfahren wenig zu einer langfristigen Gewichtsreduktion beizutragen vermögen. Nur ca. $5 \%$ aller Betroffenen können nach einer Gewichtsreduktion das erreichte Gewicht mindestens fünf Jahre halten. ${ }^{48}$

Gut belegt ist dagegen, dass bei Menschen mit extremer Adipositas in der Regel bereits moderate Gewichtsabnahmen mit einer signifikanten Besserung von Begleiterkrankungen (Typ-2-Diabetes, Hypertonie, Störungen des Fettstoffwechsels, obstruktives Schlafapnoe-Syndrom) einhergehen. ${ }^{49}$ Angesichts der hierzu existierenden Datenlage spiegelt sich möglicherweise eine Form institutioneller Stigmatisierung in der Vorenthaltung von Behandlungen wider. So haben in der Vergangenheit wiederholt Krankenkassen - nach Scheitern konventioneller Therapien - die Kostenübernahme für die Durchführung leitliniengestützter chirurgischer Interventionen („Gastric banding“) bei Patienten mit extremer Adipositas mit der Begründung verweigert, dass es sich bei der Adipositas nicht um eine Krankheit handele. In einem Grundsatzurteil hat das Bundessozialgericht am 19. Februar 2003 allerdings mittlerweile diese Auffassung der Krankenkassen zurückgewiesen (Az B1 KR 1/o2) und betont, dass im Einzelfall anhand der medizinischen Leitlinien geprüft werden muss ob, in welcher Form und in welchem Umfang Behandlungsmaßnahmen bei adipösen Patienten zulasten der gesetzlichen Krankenversicherung durchgeführt werden können.

Ungeklärt ist allerdings, ob wirklich alle übergewichtigen Menschen von einer Gewichtsabnahme profitieren. Aktuellen Daten einer finnischen Langzeitstudie mit knapp 3000 Teilnehmern zufolge ${ }^{50}$ war für moderat übergewichtige, aber ansonsten gesunde Menschen, die innerhalb eines 18-jährigen Beobachtungszeitraums erfolgreich eine Diät durchgeführt hatten, dass Sterberisiko fast doppelt so hoch wie bei den Teilnehmern mit stetem, moderaten Übergewicht.

46 z. B. Lamerz et al. (2005).

47 Birgit Herden, „Dick bleibt dick“, Die Zeit Nr. 13 vom 22.03.2007.

48 Hebebrand et al. (2004).

49 Deutsche Adipositas-Gesellschaft (2007).

50 Sørensen et al. (2005). 


\title{
5 Stigmatisierung im Zusammenhang mit Anorexia nervosa
}

Stigmatisierende Einstellungen in der Öffentlichkeit bezüglich der Anorexia nervosa finden sich - ähnlich wie bei Suchterkrankungen - vor allem in $\mathrm{Zu}$ schreibungen an die Betroffenen wie: „sind selbst schuld an der Erkrankung“ oder „müssten sich mehr zusammenreißen“. ${ }^{51}$ Im Vergleich zu Schizophrenie oder Asthma wird bei Anorexia nervosa den Betroffenen außerdem signifikant häufiger unterstellt, mit ihrer Erkrankung „Aufmerksamkeit erzielen zu wollen". . $^{2}$

Anorektisch erkrankte junge Frauen berichten selbst häufig, sich im Rahmen ihrer Erkrankung zunehmend missverstanden und isoliert zu fühlen und von ihrer Umwelt nicht mehr als individuelle Person, sondern nur noch reduziert unter den Aspekten von Gewichtsverlust oder -zunahme wahrgenommen zu werden. ${ }^{53}$ Entgegen weit verbreiteten Annahmen einer fehlenden Krankheitseinsicht nehmen viele, auch junge Patientinnen zahlreiche Nachteile der Erkrankung sehr genau wahr und können diese auch beschreiben, wie der nachfolgende Brief einer 15-jährigen Jugendlichen während der stationären Behandlung in unserer Klinik ,an die Anorexie als schlimmste Feindin“ zeigt:

\begin{abstract}
„Du bist meine schlimmste Feindin, weil ... du mir mein Leben zerstört hast/kaputtgemacht hast ... ich wegen dir mich zum Hungern zwingen musste (am Anfang) ... Ich durch dich oftmals schlecht gelaunt bin ... Ich durch dich gefroren habe ... Du mir viele Ängste und Zwänge sowie Probleme gebracht hast ... Ich mich mit vielen anderen vergleiche und sie beneide ... Ich nicht mehr richtig essen kann ... Meine Haut rau geworden ist ... Mein Lebenssinn weg ist ... Durch dich viele Tränen gelaufen sind ... Du eine Belastung für meine Familie und mich bist.“
\end{abstract}

Mit der ersten Phase der Erkrankung und Gewichtsabnahme sind oft positive Reaktionen, etwa Anerkennung oder sogar Bewunderung durch Freundinnen oder Familienmitglieder verbunden. Im Verlauf der Gewichtsabnahme entwickelt sich im Zusammenhang mit den hungerbedingten psychischen und körperlichen Veränderungen aber eine Negativ-Spirale mit immer stärkerer, kognitiver Einengung auf die Themen „Essen und Cewicht“, zwanghaften Beschäftigungen und zunehmend depressiver Stimmungslage, in deren Verlauf sich die erkrankten jungen Frauen mehr und mehr aus ihrer bisherigen sozialen Umwelt entfernen. Eindrücklich beschreibt Fechner (2007) in ihrem autobiografischen Bericht über ihre Adoleszenz mit Magersucht und Bulimie:

„Und unsere Umwelt nimmt uns das - aus Bequemlichkeitsgründen? Aus Unsicherheit? - ab. Sie glaubt uns in einer Welt, in der wir längst nicht mehr leben. Wir leben in unserer eigenen Welt, unserer Essstörungswelt. Wir versuchen, uns für dieselben Dinge zu interessieren wie die Menschen um uns herum, für Politik, Kultur, Partys, Reisen. Aber tatsächlich ist es uns egal, welchen Kinofilm man unbedingt gesehen haben muss! ... Unsere Gedanken drehen

51 Crisp et al. (2000); Stewart/Keel/Schiavo (2005).

52 Stewart/Keel/Schiavo (2005).

53 Rich (2006). 
sich um viel schwer wiegender Probleme: wieso habe ich heute auf der Waage $200 \mathrm{~g}$ mehr als gestern, muss ich meine Kalorienration noch etwas herabsetzen? Welchen Lightjoghurt esse ich in der Mittagspause: Banane ( $83 \mathrm{kcal}$ ), Kirsch (79 kcal) oder Erdbeere ( $85 \mathrm{kcal})$ ? Was mache ich, wenn es im Supermarkt keinen frischen Salat mehr gibt? ${ }^{454}$

Andere Jugendliche schildern, wie verunsicherte Eltern von Freundinnen deren Umgang mit ihnen zu reduzieren versuchen aus Angst vor einer „Ansteckung“ der eigenen Tochter mit der Magersucht. Zusätzlich erleben sie Unverständnis (,die braucht doch nur zu essen!“) und Misstrauen (,wenn ich Bauchschmerzen habe, heißt es gleich: du willst nicht essen, weil du abnehmen willst"). Möglicherweise besteht zwischen der oft lange vorherrschenden Überzeugung der jungen Frauen, „alles unter Kontrolle zu haben“, und Zuschreibungen der sozialen Umwelt, die Betroffenen seien „selbst schuld an ihrer Erkrankung" ein enger Zusammenhang, der zum Scheitern des Dialogs mit Familie und Freunden beiträgt.

In dem Maße, in dem es Eltern und Freundinnen misslingt, sich in die Welt der anorektischen Jugendlichen einzufühlen, finden diese emotionalen Rückhalt bei anderen Betroffenen, oft in „Chat-Rooms“ in Internetforen. Neben deutlichen Gefahren durch so genannte „Pro-Ana “-Seiten, auf denen die Essstörung als „Life-Style“ idealisiert wird und konkrete Anleitungen und Tipps ausgetauscht werden, die Anorexie zu erzeugen oder zu verstärken, finden sich in anderen Foren, ${ }^{55}$ aber auch sehr konstruktive, unterstützende Beiträge der Teilnehmerinnen.

Obwohl in der Vergangenheit viele der bei Anorexie-Patientinnen zu beobachtenden Verhaltensweisen, wie etwa das extrem langsame und ritualisierte Essverhalten, im Rahmen eines Hungerexperiments auch bei gesunden, jungen Männern ausgelöst werden konnten,$^{56}$ wird ein solches Verhalten bei einer anorektischen Patientin meist dahingehend interpretiert, dass diese eben weiterhin abnehmen wolle, nicht therapiemotiviert oder nicht krankheitseinsichtig sei. Dies gilt auch für den bei vielen Patientinnen mit Anorexia nervosa häufig trotz ausgeprägter Kachexie zu beobachtenden extremen Bewegungsdrang. Im Tiermodell konnte inzwischen gezeigt werden, dass Ratten bei Futterrestriktion ihr Aktivitätsniveau auf ca. 300 bis $400 \%$ des Ausgangswertes steigern und das Zustandekommen dieser Hyperaktivität durch exogene Zufuhr des Neuropeptids Leptin verhindert werden kann. ${ }^{57}$ Inzwischen konnte auch bei Patientinnen mit Anorexia nervosa ein Zusammenhang zwischen dem Ausmaß der körperlichen Aktivität und Leptinspiegeln im Serum gezeigt werden. ${ }^{58}$ Möglicherweise handelt es sich bei dieser semi-starvationsinduzierten Hyperaktivität um eine phylogenetische Reaktionsweise, zum Beispiel im Zusammenhang mit der Nahrungssuche. ${ }^{59}$ Die Vermittlung von Wissen über derartige biologische Folgen des Hungerprozesses an die Patienten selbst, aber

54 Fechner (2007), S. 63.

55 z. B. www.Magersucht.de; www.hungrig-online.de

56 Keys et al. (1950).

57 Exner et al. (2000).

58 Holtkamp et al. (2006).

59 Hebebrand et al. (2007). 
auch an deren Angehörige und Mitarbeiter des Behandlungsteams kann nach unseren klinischen Erfahrungen dazu beitragen, die verbreitete Überzeugung, dass solche Verhaltensweisen vollständig unter der willentlichen Kontrolle der Patientinnen stehen, zu hinterfragen. Die Patientinnen berichten im Nachhinein häufig, dass sie diesen Bewegungsdrang als quälend und zwanghaft erlebt haben. Möglicherweise kann durch frühzeitigere Ermöglichung dosierter Aktivität im Rahmen der Behandlung diesem Bewegungsdrang angemessener begegnet und die Bereitschaft der Patientinnen zur Mitarbeit in der Behandlung erhöht werden.

\section{Stigmatisierung der Eltern essgestörter Patientinnen}

Trotz der zunehmenden Hinweise auf genetische und andere biologische Faktoren in der Ätiologie ${ }^{60}$ werden in der Öffentlichkeit im Vergleich mit Schizophrenie und Asthma signifikant weniger biologische Faktoren, sondern in erster Linie soziale, elterliche und individuelle Faktoren als verantwortlich für die Entstehung der Anorexia nervosa vermutet. ${ }^{61}$ Dies spiegelt möglicherweise wider, dass im zeitlichen Zusammenhang mit der Entwicklung der Familientherapie in den 197oer Jahren solchen Faktoren, zum Beispiel dysfunktionalen familiären Interaktionen wie „überprotektives Verhalten“ oder „Verstrickung““62 eine ursächliche Bedeutung zugeschrieben worden war. Die damaligen Hypothesen beruhten weitgehend auf der klinischen Arbeit mit anorektischen Jugendlichen und deren Familien, ohne ausreichend $\mathrm{zu}$ berücksichtigen, dass sich möglicherweise durch die zum Teil bereits über viele Monate bestehende Essstörung die familiären Interaktionen signifikant verändert hatten. In vielen Familien kommt es zu starken emotionalen Reaktionen durch die Entwicklung einer Essstörung. Solche Reaktionen umfassen Angst und Schuldgefühle, Verzweiflung, Resignation und Wut. ${ }^{63}$ Mit der Diagnose einer Essstörung sind auf Seiten der Eltern häufig Schuldgefühle und Scham verbunden. Nicht selten bestehen auch Selbstvorwürfe, die Krankheit zu spät erkannt zu haben bzw. zu spät professionelle Hilfe gesucht zu haben. ${ }^{64}$ Spätere Studien, in denen familiäre Variablen für den Zeitraum vor Beginn einer Essstörung erfasst wurden, ermittelten zwar Unterschiede in der familiären Interaktion gegenüber einer gesunden Vergleichsgruppe, nicht aber gegenüber Kontrollgruppen mit anderen psychiatrischen Erkrankungen. ${ }^{65}$

Trotz fehlender empirischer Evidenz für eine ursächliche Bedeutung der Familie für die Entstehung der Anorexia nervosa sowie nachgewiesener Wirksamkeit der Einbeziehung der Familie in die Therapie ${ }^{66}$ erleben sich viele Eltern als ausgeschlossen von der Behandlung und nicht ernst genommen. ${ }^{67}$ In den

\footnotetext{
60 Übersicht bei Jacobi et al. (2004).

61 Stewart/Keel/Schiavo (2005).

62 Minuchin et al. (1975); Selvini-Palazolli (1974).

63 Goldner/Birmingham (1994).

64 Treasure et al. (2001); Perednia/Vandereycken (1989).

65 Webster/Palmer (2000); Fairburn et al. (1999).

66 Eisler et al. (1997), (2000).

67 McMaster et al. (2004).
} 
an unserer Klinik seit vielen Jahren durchgeführten Psychoedukationsgruppen für Eltern von Jugendlichen mit Essstörungen ${ }^{68}$ ist eine der häufigsten Klagen der Eltern die Schwierigkeit, nach Wahrnehmung von Symptomen einer Essstörung bei ihrer Tochter kompetente, professionelle Unterstützung zu finden. Viele Eltern berichten über zu lange Wartezeiten und den Eindruck, dass ihre Sorgen und Befürchtungen hinsichtlich der Entwicklung einer Essstörung bei ihrem Kind anfänglich von den aufgesuchten Ärzten und Psychotherapeuten nicht ernst genug genommen wurden: „Das legt sich wieder, warten Sie ...", „Sie machen sich zu viele Sorgen ...”, „Sie sind zu überbehütend, lassen sie Ihrer Tochter mehr Freiheiten ...", „Mischen Sie sich weniger ins Essen ein“. Dies scheint besonders dann eine Rolle zu spielen, wenn die Jugendliche noch kein deutlich erkennbares Untergewicht zeigt, aber bereits deutliche Essstörungssymptome (restriktives Essen, Gewichtsphobie) für die Eltern erkennbar sind.

Die Suche der Eltern nach Informationen über die Anorexia nervosa in Ratgebern oder im Internet kann das Gefühl, am Verhalten des Kindes schuld zu sein, verstärken. So berichtete ein Vater, wie er sich bemüht habe, sein Verhalten entsprechend dem Versuch, nicht „überprotektiv“ zu sein, zu ändern, durch die immer schlechtere körperliche Verfassung seiner 16-jährigen Tochter gleichzeitig aber immer verzweifelter geworden sei:

„M. hat damals bei einer Größe von 1,66 m nur noch 35 Kilo gewogen. Sie ist jeden Tag mit dem Fahrrad 30 Kilometer und mehr gefahren. Weil ich Angst hatte, dass sie irgend wo zusammenbricht, bin ich jedes Mal hinter ihr hergefahren. Einerseits habe ich mich geschämt, weil ich mein Verhalten als überbehütend empfand, andererseits konnte ich nicht anders!"

Die Tochter hatte eine von den Eltern seit mehreren Wochen für notwendig gehaltene stationäre Behandlung in unserer Klinik vehement abgelehnt und angedroht, sich im Falle einer Klinikaufnahme zu suizidieren. Der von der Familie aufgesuchte Psychotherapeut hatte den Eltern geraten abzuwarten, da nach seiner Meinung eine Therapie ohne Motivation der Jugendlichen keinen Erfolg haben werde.

Die Sorge vor Stigmatisierung kann auch zu Widerständen führen, mit Freunden oder Verwandten über die Erkrankung zu sprechen. Zudem äußern Eltern anorektischer Patientinnen ihren Eindruck, dass andere Menschen die Erkrankung tendenziell unterschätzen. ${ }^{69}$ Als stigmatisierende Erfahrung beschreiben sie, dass fremde Menschen auf der Straße anhalten und ihre Tochter anstarren würden. Innerhalb der Familie - besonders von Seiten der eigenen Eltern - erleben sie Vorwürfe, z. B. als Mutter durch die Wiederaufnahme der Berufstätigkeit an der Erkrankung der Tochter schuld zu sein. Vor allem die Mütter reagieren auf die Erkrankung mit heftigen emotionalen Belastungen, Schuldgefühlen und Selbstvorwürfen, sich inadäquat verhalten oder versagt zu haben. Nach Teilnahme an der in unserer Klinik durchgeführten Gruppen-

68 Hagenah et al. (2003).

69 Whitney et al. (2005). 
Psychoedukation beschreiben Eltern neben der ausführlichen, sachlichen Information zu den Ursachen und Behandlungsmöglichkeiten der Anorexia nervosa besonders die Teilnahme anderer Eltern als hilfreich und entlastend. ${ }^{70}$

\section{Zusammenfassung und Ausblick}

Sowohl für die Anorexia nervosa wie auch die Adipositas lassen sich Stigmatisierungsprozesse mit erheblichen psychosozialen Folgen für die Betroffenen, aber auch ihre Familien aufzeigen, deren Auswirkungen derzeit nicht hinreichend untersucht sind. Derartige Prozesse stellen Barrieren beim frühzeitigen Aufsuchen professioneller Hilfe dar und tragen so zur Chronifizierung bei. Stigmatisierende Einstellungen können nicht nur bei Laien, sondern auch Ärzten, Psychotherapeuten und anderen professionellen Helfern nachgewiesen werden. Das mit der Erkrankung verbundene Stigma wird nicht selten von den Betroffenen geteilt (Selbst-Stigmatisierung) und vergrößert auf diesem Weg die Folgen. Bessere Kenntnisse über die komplexen Entstehungsbedingungen von Essstörungen und Adipositas können zur Verringerung negativer Etikettierungen, „Angst vor Ansteckung“ und daraus resultierenden Berührungsängsten beitragen. Teilnehmer einer in unserer Klinik durchgeführten Gruppen-Psychoedukation für Eltern von Kindern und Jugendlichen mit Anorexia nervosa berichten immer wieder, dass die Informationen zu Erkrankung und Behandlung sowie die Teilnahme insgesamt ihnen geholfen habe, besser zwischen den Krankheitssymptomen der Essstörungen und ihrem Kind als Person unterscheiden zu können und dadurch zu einer besseren Beziehung zu ihrem Kind zurückgefunden zu haben.

Aufklärungskampagnen in den Medien, z. B. gegen das Übergewicht, können unbeabsichtigt zur Zunahme diskriminierender Einstellungen führen, wenn sie die individuelle Eigenverantwortung zu stark gewichten. Am Entstehen solcher Kampagnen sind zum Teil sehr unterschiedliche Interessengruppen beteiligt. So sind in der 2004 von der damaligen Ernährungsministerin Renate Künast ins Leben gerufenen Plattform für Ernährung und Bewegung (peb) neben medizinischen Fachgesellschaften und Sportverbänden auch der Bundesverband der Deutschen Süßwarenindustrie und zahlreiche Firmen, die mit kalorienreichen Getränken und „Fast-food“-Produkte erheblichen Umsatz durch Kinder und Jugendliche machen, vertreten. ${ }^{71}$ Im Gründungsprogramm der Plattform „Im Gleichgewicht - für ein gesundes Leben“ (peb, 2004) findet sich wenig überraschend nicht ein einziger Hinweis auf die Bedeutung dieser Produkte für die Entwicklung von Übergewicht, vielmehr wird die individuelle Eigenverantwortung (Kinder, Eltern, professionelle Berufsgruppen) hervorgehoben!

Letztlich erfordert die Auseinandersetzung mit Anorexia nervosa und Adipositas auch von Medizin und Psychotherapie das Eingeständnis, derzeit zu wenig über effektive Behandlungsverfahren zu verfügen. Dies beinhaltet die Gefahr, bei zu hohem Erwartungsdruck in Richtung einer schnellen und an-

70 Hagenah/Vloet (2005).

71 www.ernaehrung-und-bewegung.de. 
dauernden Veränderung die Gründe für das Scheitern der Behandlung einseitig den Patienten und ihren Angehörigen zuzuschreiben. Die Vermeidung von Kränkungen und Enttäuschungen auf beiden Seiten (Arzt und Patient) gelingt eher bei Vereinbarung realistischer Ziele unter Anerkennung der Tatsache, dass sowohl Anorexia nervosa auf der einen Seite wie auch Adipositas auf der anderen Seite schwer beeinflussbare, komplexe biopsychosoziale Prozesse darstellen.

Bei aller Bedeutung eines erfolgreichen „Abnehmens“ oder „Zunehmens“ für die langfristige körperliche und psychische Gesundheit darf sich der therapeutische Dialog nicht ausschließlich an diesem Fokus „festbeißen“. Gerade wenn hier ärztlich bzw. gesellschaftlich erwünschte Fortschritte nicht erreicht werden können, benötigen die betroffenen Menschen Unterstützung und Hilfe bei der Bewältigung alltäglicher Belastungen. Dazu gehört eine wertschätzende Haltung gegenüber den Anstrengungen der Patienten, wie sie anderen somatisch schwer kranken Menschen selbstverständlich zugestanden wird. Nicht unterschätzt werden darf in diesem Prozess das Phänomen der SelbstStigmatisierung mit den Auswirkungen auf die soziale Integration. (Fazit einer 19-jährigen Patientin: „Außenstehende denken, wer eine Essstörung hat, muss einen psychischen Defekt haben ...“.) Als wichtige Ressource bei der Reduktion von Stigmatisierungsfolgen haben sich bei anderen körperlichen und psychischen Erkrankungen Selbsthilfeorganisationen erwiesen, die zur Zeit allerdings weder bei Adipositas noch bei Anorexia nervosa bundesweit existiert zu sein scheinen (mit Ausnahme der Adipositaschirurgie-SelbsthilfeDeutschland e.V.). ${ }^{72}$

\section{Literatur}

Barney et al. (2006): Lisa J. Barney, Kathleen M. Griffiths, Anthony F. Jorm et al., Stigma about depression and its impact on help-seeking intentions, Australian and New Zealand Journal of Psychiatry 40 (2006), p. 51-54

Bergmann/Mensink (1999): Karl E. Bergmann, Gert B. Mensink, Körpermaße und Übergewicht, Gesundheitswesen 61 (Sonderheft) (1999), S. 115-120

Brickman (1975): Philip Brickman, Adaptation level determinants of satisfaction with equal and unequal outcome distributions in skill change situations, Journal of Personality and Social Psychology 32 (1975), p. $191-198$

Bruch (1962): Hilde Bruch, Perceptual and conceptual disturbances in anorexia nervosa, Psychosomatic Medicine 24 (1962), p. 187-194

Coon/Tucker (2002): Katharine A. Coon, Katherine L. Tucker, Television and children's consumption patterns. A review of the literature, Minerva Pediatrica 54 (2002), p. 423-436

Cramer/Steinwert (1998): Phebe Cramer, Tiffany Steinwert, Thin is good, fat is bad: How early does it begin? Journal of Applied Developmental Psychology (1998), p. 429-451

Crisp et al. (2000): Arthur H. Crisp, Michael G. Gelder, Susannah Rix et al., Stigmatisation of people with mental illnesses, British Journal of Psychiatry 177 (2000), p. 4-7

Currin et al. (2005): Laura Currin, Ulrike Schmidt, Janet Treasure et al., Time trends in eating disorder incidence, British Journal of Psychiatry 186 (2005), p. 132-135

Dejong (1993): William Dejong, Obesity as a characterological stigma: the issue of responsibility and judgements of task performance, Psychological Reports 73 (1993), p. 963-970

72 www.acsdev.de. 
Deutsche Adipositas-Gesellschaft (2007): Deutsche Adipositas-Gesellschaft, Evidenzbasierte Leitlinie: Prävention und Therapie der Adpositas, www.adipositas-gesellschaft.de

Edmunds (2005): Laurel D. Edmunds, Parents' perceptions of health professionals' responses when seeking help for their overweight children, Family Practice 22 (2005), p. 287-292

Eisler et al. (1997): Ivan Eisler, Christopher Dare, Gerald F. Russell et al., Family and individual therapy in anorexia nervosa. A 5-year follow-up, Archives of General Psychiatry 54 (1997), p. 1025-1030

Eisler et al. (2000): Ivan Eisler, Christopher Dare, Matthew Hodes et al., Family therapy for adolescent anorexia nervosa: the results of a controlled comparison of two family interventions, Journal of Child Psychology and Psychiatry and Allied Disciplines 41 (2000), p. 727-736

Epstein et al. (1994): Leonard H. Epstein, Alice Valoski, Rena R. Wing et al., Ten-year outcomes of behavioral family-based treatment for childhood obesity, Health Psychology 13 (1994), p. 373-383

Exner et al. (2000): Cornelia Exner, Johannes Hebebrand, Helmut Remschmidt et al., Leptin suppresses semistarvation induced hyperactivity in rats: implications for anorexia nervosa, Molecular Psychiatry 5 (2000), p. 476-481

Fairburn et al. (1999): Christopher G. Fairburn, Zafra Cooper, Helen A. Doll et al., Risk factors for anorexia nervosa: three integrated case-control comparisons, Archives of General Psychiatry 56 (1999), p. 468-476

Fairburn/Harrison (2003): Christopher G. Fairburn, Paul J. Harrison, Eating disorders, Lancet 361 (2003), p. 407-416

Fechner (2007): Annika Fechner, Hungrige Zeiten mit Magersucht und Bulimie, München 2007

Gaebel/Zäske/Baumann (2004): Wolfgang Gaebel, Harald Zäske, Anja Baumann, Stigma erschwert Behandlung und Integration, Deutsches Ärzteblatt 101 (2004), S. A3253-A3255

Goffman (1963): Erving Goffman, Stigma: Notes on the Management of Spoiled Identity, New York 1963

Goldner/Bimingham (1994): Elliot M. Goldner, Birmingham C. Laird, Anorexia nervosa: methods of treatment, in: LeeAnn Alexander-Mott, D. Barry Lumsden (ed.), Understanding Eating Disorders: Anorexia Nervosa, Bulimia Nervosa, and Obesity, Washington 1994, p. 135-157

Gortmaker et al. (1993): Steven L. Gortmaker, Aviva Must, James M. Perrin et al., Social and economic consequences of overweight in adolescence and young adulthood, New England Journal of Medicine 329 (1993), p. 1008-1012

Gunewardene et al. (2001): Anoushka Gunewardene, Gail F. Huon, Richang Zheng, Exposure to westernization and dieting: a cross-cultural study, International Journal of Eating Disorders 29 (2001), p. 289-293

Hagenah et al. (2003): Ulrich Hagenah, Varinja Blume, Marlene Flacke-Redanz et al., Psychoedukation als Gruppenangebot für Eltern essgestörter Jugendlicher, Zeitschrift für Kinder- und Jugendpsychiatrie und Psychotherapie 31 (2003), S. 51-58

Hagenah/Vloet (2005): Ulrich Hagenah, Timo Vloet, Psychoedukation für Eltern in der Behandlung essgestörter Jugendlicher, Praxis der Kinderpsychologie und Kinderpsychiatrie 54 (2005), S. 303-317

Haghighat (2001): Rahman Haghighat, Towards a unitary theory of stigmatisation, British Journal of Psychiatry 178 (2001), p. $378 \mathrm{f}$.

Hebebrand et al. (2004): Johannes Hebebrand, Peter Dabrock, Michael Lingenfelder et al., Ist Adipositas eine Krankheit? Interdisziplinäre Perspektiven, Deutsches Ärzteblatt 101 (2004), S. A2468-A2474

Hebebrand et al. (2007): Johannes Hebebrand, Timo D. Müller, Kristian Holtkamp et al., The role of leptin in anorexia nervosa: clinical implications, Molecular Psychiatry 12 (2007), p. 23-35

Herpertz-Dahlmann et al. (2003): Beate Herpertz-DahImann, Frank Geller, Corinna Bohle et al., Secular trends in body mass index measurements in preschool children from the City of Aachen, Germany, European Journal of Pediatrics 162 (2003), p. 104-109

Hoek (2006): Hans Wijbrand Hoek, Incidence, prevalence and mortality of anorexia nervosa and other eating disorders, Current Opinion in Psychiatry 19 (2006), p. 389-394

Holtkamp et al. (2006): Kristian Holtkamp, Beate Herpertz-Dahlmann, Kathrin Hebebrand et al., Physical activity and restlessness correlate with leptin levels in patients with adolescent anorexia nervosa, Biological Psychiatry 60 (2006), p. 311-313

Jacobi et al. (2004): Corinna Jacobi, Chris Hayward, Helena C. Kraemer et al., Coming to terms with risk factors for eating disorders: application of risk terminology and suggestions for a general taxonomy, Psychological Bulletin 130 (2004), p. 19-65 
Keys et al. (1950): Ancel Keys, Josef Brozek, Austin Henschel et al., The Biology of Human Starvation, Minneapolis 1950

Lamerz et al. (2005): Andreas Lamerz, Jutta Kuepper-Nybelen, Christine Wehle et al., Social class, parental education, and obesity prevalence in a study of six-year-old children in Germany, International Journal of Obesity 29 (2005), p. 373-380

Latner/Stunkard (2003): Janet D. Latner, Albert J. Stunkard, Getting worse: the stigmatization of obese children, Obesity Research 11 (2003), p. 452-456

Latner/Stunkard/Wilson (2005): Janet D. Latner, Albert J. Stunkard, G. Terence Wilson, Stigmatized students: age, sex, and ethnicity effects in the stigmatization of obesity, Obesity Research 13 (2005), p. 1226-1231

Link/Phelan (2001): Bruce G. Link, Jo C. Phelan, Conceptualizing Stigma, Annual Review of Sociology 27 (2001), p. $363-385$

Lucas et al. (1999): Alexander R. Lucas, Cynthia S. Crowson, W. Michael O'Fallon et al., The ups and downs of anorexia nervosa, International Journal of Eating Disorders 26 (1999), p. 397-405

Makino/Tsuboi/Dennerstein (2004): M. Makino, K. Tsuboi, L. Dennerstein, Prevalence of eating disorders: a comparison of Western and non-Western countries, Medscape general medicine 6 (2004), p. 49

McMaster et al. (2004): Rose McMaster, Barbara Beale, Sharon Hillege et al., The parent experience of eating disorders: interactions with health professionals, International Journal of Mental Health Nursing 13 (2004), p. 67-73

Minuchin et al. (1975): Salvador Minuchin, Lester Baker, Bernice L. Rosman et al., A conceptual model of psychosomatic illness in children. Family organization and family therapy, Archives of General Psychiatry 32 (1975), p. 1031-1038

Müller (1996): Klaus E. Müller, Der Krüppel: Ethnologia passionis humanae, München 1996

Myers/Rosen (1999): Anna Myers, James C. Rosen, Obesity stigmatization and coping: relation to mental health symptoms, body image, and self-esteem, International Journal of Obesity 23 (1999), p. 221-230

Nasser (1993): Mervat Nasser, A prescription of vomiting: historical footnotes, International Journal of Eating Disorders 13 (1993), p. 129-131

Nicholls/Viner (2005): Dasha Nicholls, Russell Viner, Eating disorders and weight problems, British Medical Journal 330 (2005), p. 950-953

Ogden et al. (2002): Cynthia L. Ogden, Katherine M. Flegal, Margaret D. Carroll et al., Prevalence and trends in overweight among US children and adolescents, 1999-2000, JAMA 288 (2002), p. 1728-1732

Patton et al. (1990): Gregory C. Patton, Eric Johnson-Sabine, Ken Wood et al., Abnormal eating attitudes in London schoolgirls - a prospective epidemiological study: outcome at twelve month follow-up, Psychological Medicine 20 (1990), p. 383-394

Perednia/Vandereycken (1989): Claire Perednia, Walter Vandereycken, An explorative study on parenting in eating disorder families, in: Walter Vandereycken, Elly Kog, Johan Vanderlinden (ed.), The Family Approach to Eating Disorders, New York 1989, p. 119-146

Petrovich et al. (2005): Gorica D. Petrovich, Peter C. Holland, Michaela Gallagher, Amygdalar and prefrontal pathways to the lateral hypothalamus are activated by a learned cue that stimulates eating, Journal of Neuroscience 25 (2005), p. 8295-8302

Pierce/Wardle (1997): Jaqueline W. Pierce, Jane Wardle, Cause and effect beliefs and self-esteem of overweight children, Journal of Child Psychology and Psychiatry and Allied Disciplines 38 (1997), p. 645-650

Peb (2004): Plattform für Ernährung und Bewegung (peb), Gründungsprogramm „Im Gleichgewicht - für ein gesundes Leben“, www.ernaehrung-und-bewegung.de

Price et al. (1987): James H. Price, Sharon M. Desmond, Ronald A. Krol et al., Family practice physicians' beliefs, attitudes, and practices regarding obesity, American Journal of Preventive Medicine 3 (1987), p. 339-345

Raimbault/Eliacheff (1989): Ginette Raimbault, Caroline Eliacheff, Les indomptables figures de l'anorexie, Paris 1989

Rich (2006): Emma Rich, Anorexic dis(connection): managing anorexia as an illness and an identity, Sociology of Health and Illness 28 (2006), p. 284-305

Saper/Chou/Elmquist (2002): Clifford B. Saper, Thomas C. Chou, Joel K. Elmquist, The need to feed: homeostatic and hedonic control of eating, Neuron 36 (2002), p. 199-211

Selvini-Palazzoli (1974): Mara Selvini-Palazzoli, Self-Starvation: From the Intrapsychic to the Transpersonal Approach to Anorexia Nervosa, London 1974 
Sørensen et al. (2005): Thorkild I. Sørensen, Aila Rissanen, Maarit Korkeila et al., Intention to lose weight, weight changes, and 18-y mortality in overweight individuals without co-morbidities, PLoS Medicine 2 (2005), 6, p. e171

Stewart/Keel/Schiavo (2006): Maria-Christiana Stewart, Pamela K. Keel, R. Steven Schiavo, Stigmatization of anorexia nervosa, International Journal of Eating Disorders 39 (2006), p. 320-325

Stroessner/Mackie (1993): Steven I Stroessner, Diane M. Mackie, Affect and perceived group variability: implications for stereotyping and prejudice, in: Diane M. Mackie, David L Hamilton (ed.), Affect, Cognition, and Stereotyping: Interactive Processes in Group Perception, San Diego, CA 1993, p. 63-86

Szczypka et al. (2001): Mark S. Szczypka, Keith Kwok, Michelle D. Brot et al., Dopamine production in the caudate putamen restores feeding in dopamine-deficient mice, Neuron 30 (2001), p. 819-828

Taylor et al. (2006): C. Barr Taylor, Susan Bryson, Angela A. Celio Doyle et al., The adverse effect of negative comments about weight and shape from family and siblings on women at high risk for eating disorders, Pediatrics 118 (2006), p. 731-738

The Henry | Kaiser Family Foundation (2004): The Henry | Kaiser Family Foundation, The role of the media in childhood obesity, www.kff.org

Treasure et al. (2001): Janet Treasure, Tara Murphy, George Szmukler et al., The experience of caregiving for severe mental illness: a comparison between anorexia nervosa and psychosis, Social Psychiatry and Psychiatric Epidemiology 36 (2001), p. 343-347

Van Hoeken et al. (2003): Daphne van Hoeken, Jacob Seidell, Hans Wijbrand Hoek, Epidemiology, in: Janet Treasure, Ulrike Schmidt, Eric F. van Furth (ed.), Handbook of Eating Disorders, Chichester 2003, p. 11-34

Van Son et al. (2006): Gabrielle E. van Son, Daphne van Hoeken, Aad I. Bartelds et al., Time trends in the incidence of eating disorders: a primary care study in the Netherlands, International Journal of Eating Disorders 39 (2006), p. 565-569

Webster/Palmer (2000): Jenny J. Webster, Robert L. Palmer, The childhood and family background of women with clinical eating disorders: a comparison with women with major depression and women without psychiatric disorder, Psychological Medicine 30 (2000), p. 53-60

Westenhöfer/Pudel (1990): Joachim Westenhöfer, Volker Pudel, Gesellschaftliche Aspekte von Essstörungen, Praxis der klinischen Verhaltensmedizin und Rehabilitation 3 (1990), S. 151-159

Whitney et al. (2005): Jenna Whitney, Joanna Murray, Kay Gavan et al., Experience of caring for someone with anorexia nervosa: qualitative study, British Journal of Psychiatry 187 (2005), p. 444-449

\section{Internet}

www.Magersucht.de

www.hungrig-online.de

www.acsdev.de (Adipositaschirurgie-Selbsthilfe-Deutschland e.V.) 